\title{
Theoretical evaluation of the impact of finite intervals in the measurement of the bidirectional reflectance distribution function
}

\author{
Alejandro Ferrero $\mathbb{D}$
}

(C) The Author(s) 2019

\begin{abstract}
The bidirectional reflectance distribution function (BRDF) is a well-defined quantity that allows the bidirectional reflectance of surfaces to be described. However, the light propagation in a specific direction cannot be perfectly realized in practice, because the physical apertures are not infinitesimal but finite. Consequently, a BRDF measurement cannot be considered fully bidirectional, although the measure coincides with the BRDF within a certain confidence level. To properly understand the impact of the finite apertures on the BRDF measures, the deviation between the real BRDF and the BRDF to be obtained using real experimental conditions with finite apertures was theoretically studied for surfaces with realistic BRDFs. The biconical reflectance factor was used to estimate these "measured BRDFs" in different geometrical conditions, and a family of negative exponential functions was defined to assess the impact on surfaces with different angular scattering distributions. An expression for estimating the relative error from finite apertures is given, which considers the angular variation of the BRDF and the different solid angles involved in the measurement.
\end{abstract}

Keywords Reflection, BSDF, BRDF, and BTDF, Scattering measurements, Metrology

\section{Introduction}

The complete description of the reflection of the radiant flux by an elementary surface $(\mathrm{d} A)$ requires accounting for the partial radiant flux reflected $\left(\mathrm{d} \Phi_{\mathrm{r}}\right)$ in every direction $\left(\mathbf{r}_{\mathrm{r}}\right)$, which in turn depends on the

A. Ferrero $(\bowtie)$

Instituto de Óptica "Daza de Valdés", Agencia Estatal

CSIC, C/Serrano 121, 28006 Madrid, Spain

e-mail: alejandro.ferrero@csic.es incident radiant flux $\left(\mathrm{d} \Phi_{\mathrm{i}}\right)$ and its direction $\left(\mathbf{r}_{\mathrm{i}}\right)$. The first complication arises from the fact that the radiant flux within an infinitesimally specified direction is zero. This makes it impossible to define the bidirectional reflectance $\rho\left(\mathbf{r}_{\mathrm{i}} ; \mathbf{r}_{\mathrm{r}}\right)$ as the ratio between the reflected radiant flux and the incident radiant flux for given incidence and reflection directions. Let the directional radiant fluxes be defined as:

$\mathrm{d} \Phi_{\mathrm{i}}\left(\mathbf{r}_{\mathrm{i}}\right)=L_{\mathrm{i}}\left(\mathbf{r}_{\mathrm{i}}\right) \mathrm{d} A \mathrm{~d} \Omega_{\mathrm{i}}$

$\mathrm{d} \Phi_{\mathrm{r}}\left(\mathbf{r}_{\mathrm{i}} ; \mathbf{r}_{\mathrm{r}}\right)=L_{\mathrm{r}}\left(\mathbf{r}_{\mathrm{i}} ; \mathbf{r}_{\mathrm{r}} ; L_{\mathrm{i}}\right) \mathrm{d} A \mathrm{~d} \Omega_{\mathrm{r}}$

where $L_{\mathrm{i}}$ and $L_{\mathrm{r}}$ are the radiances related to the incident and reflected radiant fluxes within the irradiation and collection solid angles $\mathrm{d} \omega_{\mathrm{i}}$ and $\mathrm{d} \omega_{\mathrm{r}}$, respectively, and $\mathrm{d} \Omega_{\mathrm{i}}$ and $\mathrm{d} \Omega_{\mathrm{r}}$ are their corresponding projected solid angles, calculated as $\mathrm{d} \Omega=\mathrm{d} \omega \cos \theta, \theta$ being the angle of the incidence ( $\mathrm{i}$ subscript) or reflection ( $r$ subscript) directions with respect to the normal to elementary surface, $\mathrm{d} A$. Notice that, whereas $\mathrm{d} \Phi_{\mathrm{i}}$ only depends on the incidence direction, $\mathrm{d} \Phi_{\mathrm{r}}$ depends on both incidence and reflection directions, because the radiance obtained at the reflection direction is a function of the incidence direction. In addition, $L_{\mathrm{r}}$ also depends on $L_{\mathrm{i}}$, and it is explicitly written in equation (2).

The bidirectional reflectance expressed as:

$\rho\left(\mathbf{r}_{\mathrm{i}} ; \mathbf{r}_{\mathrm{r}}\right)=\frac{\mathrm{d} \Phi_{\mathrm{r}}\left(\mathbf{r}_{\mathrm{i}} ; \mathbf{r}_{\mathrm{r}}\right)}{\mathrm{d} \Phi_{\mathrm{i}}\left(\mathbf{r}_{\mathrm{i}}\right)}=\frac{L_{\mathrm{r}}\left(\mathbf{r}_{\mathrm{i}} ; \mathbf{r}_{\mathrm{r}} ; L_{\mathrm{i}}\right) \mathrm{d} \Omega_{\mathrm{r}}}{L_{\mathrm{i}}\left(\mathbf{r}_{\mathrm{i}}\right) \mathrm{d} \Omega_{\mathrm{i}}}$

is undetermined, since the limit:

$\lim _{\mathrm{d} \Omega_{\mathrm{r}}, \mathrm{d} \Omega_{\mathrm{i}} \rightarrow 0} \frac{\mathrm{d} \Omega_{\mathrm{r}}}{\mathrm{d} \Omega_{\mathrm{i}}}=\lim _{\mathrm{d} \omega_{\mathrm{r}}, \mathrm{d} \omega_{\mathrm{i}} \rightarrow 0} \frac{\mathrm{d} \omega_{\mathrm{r}} \cos \theta_{\mathrm{r}}}{\mathrm{d} \omega_{\mathrm{i}} \cos \theta_{\mathrm{i}}}$ 
does not exist, or, in other words: The bidirectional reflectance would depend on the geometry of the measurement, and not only on surface properties.

Fred E. Nicodemus, aware of this inconvenience, proposed an approach to define a bidirectional reflectance quantity based on the following considerations on the involved quantities: ${ }^{1}$

1. $L_{\mathrm{r}}$ is linear with respect to $L_{\mathrm{i}}$ : For given incidence and reflection directions, $L_{\mathrm{r}}$ is proportional to $L_{\mathrm{i}}$ $\left(L_{\mathrm{r}} \propto L_{\mathrm{i}}\right)$. It is fulfilled as long as the irradiation is not so high to produce nonlinear effects.

2. $L_{\mathrm{r}}$ is linear with respect to $\mathrm{d} \Omega_{\mathrm{i}}$ : For given incidence and reflection directions, $L_{\mathrm{r}}$ is proportional to $\mathrm{d} \Omega_{\mathrm{i}}$ $\left(L_{\mathrm{r}} \propto \mathrm{d} \Omega_{\mathrm{i}}\right)$, as long as " $\mathrm{d} \Omega_{\mathrm{i}}$ is chosen small enough that there is no significant difference in the radiant absorptance for all rays incident on $\mathrm{d} A$ within the solid angle $\mathrm{d} \omega_{\mathrm{i}},{ }^{, 1}$

3. $\rho$ is invariant with respect to $L_{\mathrm{i}}$ and $\mathrm{d} \Omega_{\mathrm{i}}$ : As a consequence of the two previous considerations, for given incidence and reflection directions, $\rho$, as defined in equation (3), is invariant with respect to $L_{\mathrm{i}}$ and $\mathrm{d} \Omega_{\mathrm{i}}$. Since $L_{\mathrm{r}}=f_{\mathrm{r}} L_{\mathrm{i}} \mathrm{d} \Omega_{\mathrm{i}}$ (from i and ii, where $f_{\mathrm{r}}$ is a constant for a given geometry), then $\rho=f_{\mathrm{r}} \mathrm{d} \Omega_{\mathrm{r}}$.

4. $\rho$ is linear with respect to $\mathrm{d} \Omega_{\mathrm{r}}$ : For given incidence and reflection directions, $\rho$ is proportional to $\mathrm{d} \Omega_{\mathrm{r}}$ $\left(\rho \propto \mathrm{d} \Omega_{\mathrm{r}}\right)$, which "holds only for values of $\mathrm{d} \Omega_{\mathrm{r}}$ small enough so that the partial reflectance does not change significantly with direction for rays within the solid angle $\mathrm{d} \omega_{\mathrm{r}}$."

These are the foundations of the quantity proposed by Nicodemus to measure the bidirectional reflectance. $\mathrm{He}$ correctly reasoned that, bearing in mind these considerations, the quantity representing the bidirectional reflecting properties of a surface, independent of the geometry of the solid angles used in the measurement, could be defined as the proportionality constant between $\rho$ and $\mathrm{d} \Omega_{\mathrm{r}}$ :

$f_{\mathrm{r}}\left(\mathbf{r}_{\mathrm{i}} ; \mathbf{r}_{\mathrm{r}}\right)=\frac{\rho\left(\mathbf{r}_{\mathrm{i}} ; \mathbf{r}_{\mathrm{r}}\right)}{\mathrm{d} \Omega_{\mathrm{r}}}=\frac{L_{\mathrm{r}}\left(\mathbf{r}_{\mathrm{i}} ; \mathbf{r}_{\mathrm{r}} ; L_{\mathrm{i}}\right)}{L_{\mathrm{i}}\left(\mathbf{r}_{\mathrm{i}}\right) \mathrm{d} \Omega_{\mathrm{i}}}$

Nicodemus preferred to use the differential term $\mathrm{d} L_{\mathrm{r}}$ instead of $L_{\mathrm{r}}$ because "other incident radiation (from other sources in other directions) may also be diffusely reflected (scattered) into the same reflected directions along with that from the source under consideration." What Nicodemus actually proposed was to avoid the dependence of the bidirectional reflectance $\rho$ on the reflection solid angle $\mathrm{d} \Omega_{\mathrm{r}}$ by normalizing $\rho$ by $\mathrm{d} \Omega_{\mathrm{r}}$. This new quantity, denoted by $f_{\mathrm{r}}$ in equation (5), is the well-known bidirectional reflectance distribution function (BRDF), and it is widely used nowadays in diverse fields such as remote sensing, radiometry, or 3D-rendering as the scattering function. The Optics Classification and Indexing Scheme (OCIS) has reserved a code for it, which proves its general acceptance. The monograph on reflectance published by Nicodemus et al. in $1977,{ }^{2}$ where the concept of BRDF is thoroughly explained, has been cited more than 1600 times by scientific publications in this century.

However, there are some aspects of the BRDF that are difficult to understand. Perhaps the most obvious is why the BRDF can reach any positive value, even an infinite value for perfectly specular reflectors. The reason is that $f_{\mathrm{r}}$ is defined as a division by a solid angle element [equation (5)]. The magnitude of these solid angle elements must be small enough to contain only directions at which the involved quantities (radiance or bidirectional reflectance) do not change significantly. For glossy surfaces, for which the reflectance changes rapidly approaching the specular peak, $\mathrm{d} \omega_{\mathrm{r}}$ needs to be very small in order to meet this requirement, resulting in very high BRDF values near the specular peak [equation (5)]. This partial reflectance is a Dirac delta in the case of perfectly specular reflectors, so the BRDF has no defined value in specular directions.

According to the terminology introduced by $\mathrm{Judd}^{3}$ and later adopted by Nicodemus ${ }^{2}$, three adjectives are used to describe the reflectance under general geometrical conditions of irradiation and collection: directional (flux confined to an infinitesimal solid angle), conical (flux distributed over a finite solid angle), and hemispherical (flux distributed over the full hemisphere). These forms of reflectances are obtained by integration of $f_{\mathrm{r}}\left(\mathbf{r}_{\mathbf{i}} ; \mathbf{r}_{\mathrm{r}}\right)$.

Biconical reflectance, with both irradiation and collection in conical geometry, can be regarded as the general case, and the other reflectance coefficients are obtained by using extreme values of either irradiation or collection solid angles. It is calculated as the integrated value of the function $f_{\mathrm{r}}\left(\mathbf{r}_{\mathrm{i}}, \mathbf{r}_{\mathrm{r}}\right)$ over the specified solid angles ${ }^{2}, \omega_{\mathrm{i}}$ and $\omega_{\mathrm{r}}$ :

$\rho\left(\mathbf{r}_{\mathrm{i} 0}, \omega_{\mathrm{i}} ; \mathbf{r}_{\mathrm{r} 0}, \omega_{\mathrm{r}}\right)=\frac{1}{\Omega_{\mathrm{i}}} \int_{\omega_{\mathrm{i}}} \int_{\omega_{\mathrm{r}}} f_{\mathrm{r}}\left(\mathbf{r}_{\mathrm{i}} ; \mathbf{r}_{\mathrm{r}}\right) \mathrm{d} \Omega_{\mathrm{i}} \mathrm{d} \Omega_{\mathrm{r}}$

where $\mathbf{r}_{\mathrm{i} 0}$ and $\mathbf{r}_{\mathrm{r} 0}$ are the central incidence and collection directions of the solid angles.

Similarly, the biconical reflectance factor (ratio between the reflected radiant flux and the ideally reflected radiant flux by a perfect reflecting diffuser, PRD) is given $b^{2}$ :

$R\left(\mathbf{r}_{\mathrm{i} 0}, \omega_{\mathrm{i}} ; \mathbf{r}_{\mathrm{r} 0}, \omega_{\mathrm{r}}\right)=\frac{\pi}{\Omega_{\mathrm{i}} \Omega_{\mathrm{r}}} \int_{\omega_{\mathrm{i}}} \int_{\omega_{\mathrm{r}}} f_{\mathrm{r}}\left(\mathbf{r}_{\mathrm{i}} ; \mathbf{r}_{\mathrm{r}}\right) \mathrm{d} \Omega_{\mathrm{i}} \mathrm{d} \Omega_{\mathrm{r}}$

In practice, $\omega_{\mathrm{i}}$ and $\omega_{\mathrm{r}}$ are not completely zero, and a biconical quantity, instead of a bidirectional one, is measured. Therefore, these concepts will be used in the next section to establish the relation between the BRDF and the quantity actually measured. Some of the assumptions in the BRDF definition, as described above (" $\mathrm{d} \Omega_{\mathrm{i}}$ chosen small enough that there is no significant difference in the radiant absorptance for all rays incident on $\mathrm{d} A$ within the solid angle $\mathrm{d} \omega_{\mathrm{i}}$," or 
" $\mathrm{d} \Omega_{\mathrm{r}}$ small enough so that the partial reflectance does not change significantly with direction for rays within the solid angle $\mathrm{d} \omega_{\mathrm{r}}$ "), need to be considered in the measurement of the BRDF, in order to avoid systematic errors or to account for them in the uncertainty budget. In practice, since physical irradiation and collection apertures are finite (neither infinitely big nor infinitesimal), and the measurement area on the surface has a certain size, it is irradiated with radiant fluxes from different directions, and the collected radiant flux comes from different directions too. To understand properly the limitations of a BRDF measurement, the deviation between the real BRDF and the BRDF to be obtained using real experimental conditions with finite apertures needs to be assessed.

The consequence of using a finite measurement area and finite irradiation and collection solid angles is that the BRDF is evaluated as a weighted average over a set of pairs of irradiation and collection directions, which can be modeled with the expression in equation (7). As long as this weighted average coincides with the real BRDF with a negligible deviation, the measurement conditions can be considered adequate.

The following simple examples illustrate the impact of using finite interval. Four different BRDF angular distributions normalized to the value at $\theta_{\text {asp }}=0^{\circ}$ are shown in Fig. 1, where $\theta_{\text {asp }}$ denotes the aspecular angle, which is the angular deviation between collec- tion and specular directions. For each distribution, the average BRDF at $\theta_{\text {asp }}=0^{\circ}$ was estimated by integration, with a collection solid angle subtending an angle of $\kappa=5^{\circ}$, much larger than usual to show better the effect in the graphs. For simplicity, the sizes of the measurement area and the irradiation solid angle were not considered in these illustrating examples.

The upper left plot shows a BRDF distribution without curvature. In this case, the average BRDF coincides with the value of the BRDF at $\theta_{\text {asp }}=0^{\circ}$. For this distribution, the chosen experimental condition regarding the solid angle does not limit the accuracy of the measurement. This would not be true for the distributions shown in the upper right plot and the lower left plot, which are very representative of the BRDFs of matte materials at large incidence angles. ${ }^{4}$ In both cases, there is a curvature in the distribution, and a slight deviation is observed between the average $\mathrm{BRDF}$ and the actual value of the BRDF at $\theta_{\text {asp }}=0^{\circ}$. This deviation is almost three times larger for the distribution in the upper right plot, which can be explained by a steeper slope of the distribution. For both cases, the deviation is always smaller with a smaller collection angle. Finally, the distribution shown in the lower right plot is not monotonic and has a sharp peak. This example may represent specular reflection. In this case, the deviation is quite large, and the measurement would be meaningless. Again, the devi-
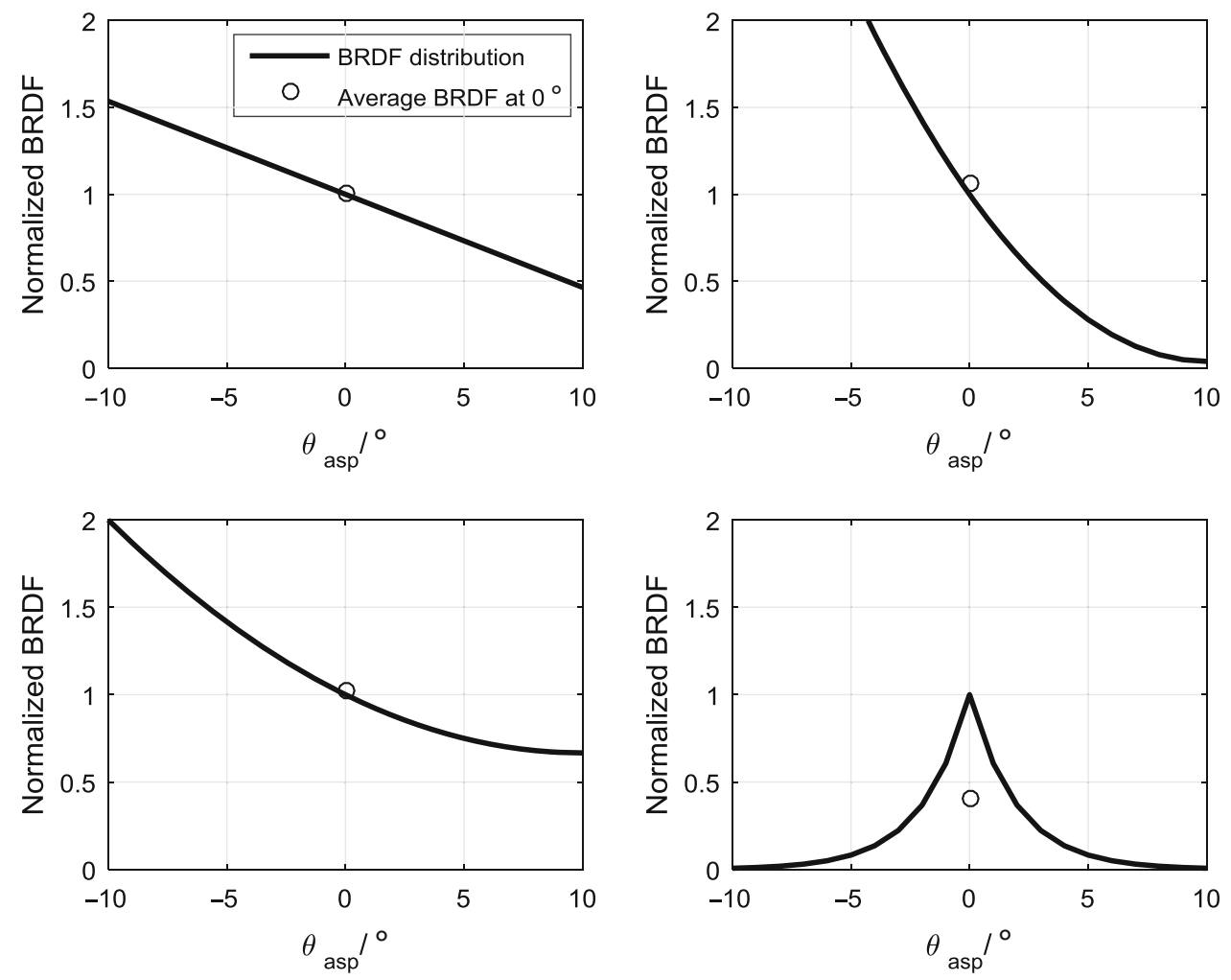

Fig. 1: Four representative BRDF distributions and the impact of a finite solid angle when measuring these distributions. The solid line represents the BRDF distribution as a function of the aspecular angle. The circle is the average BRDF over $a \pm \mathbf{5}$ degree window 
ation would always be lower using a smaller collection solid angle.

From this discussion, it can be asserted that the usage of finite apertures limits the BRDF measurements when its distribution has a curvature in the measuring angular range, being more critical when the relative variation of the distribution is higher. The maximum finite sizes of involved intervals that would give an acceptable error are what this article intends to provide. Specular reflection is an important case, but it is not the only one. Any reflectance highly nonlinear with respect to the geometry must be evaluated.

It is an important metrological issue. The International Commission on Illumination (CIE) has recently created a technical committee "Recommendation on the geometrical parameters for the measurement of the bidirectional reflectance distribution function (BRDF)" to "provide geometrical recommendations for the BRDF measurement according to the type of sample under investigation, in order to allow better comparison between the different instruments, to improve the traceability of the measurements, and to help the user to choose the right angular configuration." A description of the metrological issues to be considered in the BRDF measurement around the specular peak was published, ${ }^{5}$ and it is a very good reference to understand the problem in practice.

This work examines the impact of using finite intervals in the measurement of BRDF, for BRDFs with distributions with different relative variations, and it is proposed as a practical approach to evaluate the limitations of instruments designed for the measurement of the BRDF, and the errors produced by these limitations. Only the impact due to the geometry is taken into account in this article. Out of scope are other effects from using finite areas, such as nonuniformity, which need to be separately considered.

\section{Estimation of the measured BRDF}

The value obtained when attempting to measure the BRDF of a sample at a given geometry is denoted by "measured BRDF" here. Any instrument designed for this task has irradiation and collection apertures with finite areas, and the radiant flux reflected by the sample and collected by the instrument receiver comes from a finite area: the "measurement area." These areas are graphically depicted in Fig. 2. Four angles are subtended by the irradiation, collection, and measurement areas $\left(\kappa_{\mathrm{i}}, \kappa_{\mathrm{r}}, \kappa_{\mathrm{ma}, \mathrm{i}}\right.$, and $\left.\kappa_{\mathrm{ma}, \mathrm{r}}\right)$, and all of them affect the measurement. Notice that, whereas $\kappa_{\mathrm{i}}$ and $\kappa_{\mathrm{r}}$ are constant for any pair of incidence and collection directions $\left(\theta_{\mathrm{i} 0}\right.$ and $\left.\theta_{\mathrm{r} 0}\right)$ (right cone), $\kappa_{\mathrm{ma}, \mathrm{i}}$ and $\kappa_{\mathrm{ma}, \mathrm{r}}$ can take slightly different values depending on the illumination and collection directions and the selected point at the perimeter of the measurement area (oblique cone). The values given here for $\kappa_{\mathrm{ma}, \mathrm{i}}$ and $\kappa_{\mathrm{ma}, \mathrm{r}}$ are for the geometry with $\theta_{\mathrm{i} 0}$ and $\theta_{\mathrm{r} 0}=0^{\circ}$, for

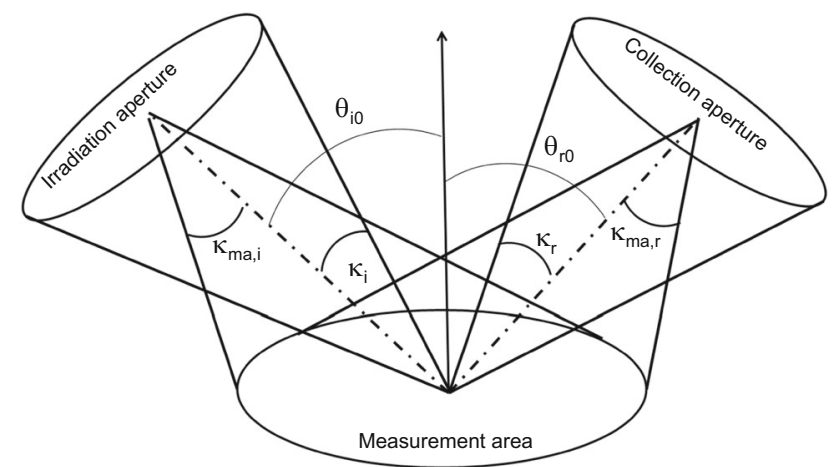

Fig. 2: Definition of the $\kappa$ angles used to describe the limitation imposed by finite intervals

which these values are independent of the selected point at the perimeter of the measurement area.

Only the pair of axial directions of the solid angles in Fig. 2 reproduces exactly the bidirectional geometry at which the BRDF is intended to be measured. The inclusion of the other pairs of directions has an impact on the measurement which depends on the sensitivity of the BRDF to geometry and to the size of the solid angles. This impact is what we want to examine here.

The measured BRDF can be estimated if the scattering distribution function and the finite intervals involved in the measurement are known. The measured BRDF, $f_{\mathrm{r}, \mathrm{m}}$, is, by definition, the biconical reflectance factor given in equation (7) divided by $\pi$. Then, the measured BRDF (for incidence and collection directions $\mathbf{r}_{\mathrm{i} 0}$ and $\mathbf{r}_{\mathrm{r} 0}$ ) can be expressed as:

$f_{\mathrm{r}, \mathrm{m}}\left(\mathbf{r}_{\mathrm{i} 0}, \omega_{\mathrm{i}} ; \mathbf{r}_{\mathrm{r} 0}, \omega_{\mathrm{r}}\right)=\frac{1}{\Omega_{\mathrm{i}} \Omega_{\mathrm{r}}} \int_{\omega_{\mathrm{i}}} \int_{\omega_{\mathrm{r}}} f_{\mathrm{r}}\left(\mathbf{r}_{\mathrm{i}} ; \mathbf{r}_{\mathrm{r}}\right) \mathrm{d} \Omega_{\mathrm{i}} \mathrm{d} \Omega_{\mathrm{r}}$

Notice that this expression does not include explicitly the measurement area. The BRDF was defined by Nicodemus et al. ${ }^{2}$ as the integration of a more complex scattering distribution function $(S)$ over an elementary area $\left(A_{\mathrm{i}}\right)$ :

$f_{\mathrm{r}}\left(\mathbf{r}_{\mathrm{i}} ; \mathbf{r}_{\mathrm{r}}\right)=\int_{\mathrm{A}} S\left(\mathbf{r}_{\mathrm{i}}, \mathbf{x}_{\mathrm{i}} ; \mathbf{r}_{\mathrm{r}}, \mathbf{x}_{\mathrm{r}}\right) \mathrm{d} A_{\mathrm{i}}$

where $\mathbf{x}_{\mathrm{i}}$ and $\mathbf{x}_{\mathrm{r}}$ are two-dimensional vectors representing the positions of the points at the surface of the incident radiant flux and the exiting radiant flux, respectively. The value of the quantity $S$ (known as bidirectional scattering-surface reflectance distribution function or BSSRDF) depends on $\mathbf{r}_{\mathrm{i}}, \mathbf{x}_{\mathrm{i}}, \mathbf{r}_{\mathrm{r}}$, and $\mathbf{x}_{\mathrm{r}}$, and it is much more complex to measure than the BRDF. For this analysis, it was assumed that the elementary area $\left(A_{\mathrm{i}}\right)$ is large enough to be considered as a measurement area and that its reflectance properties are highly uniform. In these conditions, the value of $S$ depends much more on $\mathbf{r}_{\mathrm{i}}$ and $\mathbf{r}_{\mathrm{r}}$ than on $\mathbf{x}_{\mathrm{i}}$ and $\mathbf{x}_{\mathrm{r}}$, and the latter dependence can be neglected. Notice that indistinguishable but separated points on the measurement 
area have different corresponding pairs of incidence and collection directions, which can introduce significant variations on the value of $S$.

The expression of $f_{\mathrm{r}}\left(\mathbf{r}_{\mathrm{i}}, \mathbf{r}_{\mathrm{r}}\right)$ [equation (9)] is inserted in equation (8), obtaining:

$f_{\mathrm{r}, \mathrm{m}}\left(\mathbf{r}_{\mathrm{i} 0}, \omega_{\mathrm{i}} ; \mathbf{r}_{\mathrm{r} 0}, \omega_{\mathrm{r}}\right)=\frac{1}{\Omega_{\mathrm{i}} \Omega_{\mathrm{r}}} \int_{\omega_{\mathrm{i}}} \int_{\omega_{\mathrm{r}}} \int_{\mathrm{A}} S\left(\mathbf{r}_{\mathrm{i}} ; \mathbf{r}_{\mathrm{r}}\right) \mathrm{d} A_{\mathrm{i}} \mathrm{d} \Omega_{\mathrm{i}} \mathrm{d} \Omega_{\mathrm{r}}$

where the dependence on $\mathbf{x}_{\mathrm{i}}$ and $\mathbf{x}_{\mathrm{r}}$ is already neglected.

It can be given as a function of the involved subtended angles using McCamy's notation for right circular cones: $:^{2,6}$

$$
\begin{aligned}
& f_{\mathrm{r}, \mathrm{m}}\left(\theta_{\mathrm{i} 0}, \varphi_{\mathrm{i} 0}, \kappa_{\mathrm{i}} ; \theta_{\mathrm{r} 0}, \varphi_{\mathrm{r} 0}, \kappa_{\mathrm{i}} ; R_{\mathrm{A}}\right) \\
& \quad=\frac{\int_{\theta_{i 0}-\kappa_{\mathrm{i}}}^{\theta_{\mathrm{i}}+\kappa_{\mathrm{i}}} \mathrm{d} \theta_{\mathrm{i}} \int_{\theta_{\mathrm{r} 0}-\kappa_{\mathrm{r}}}^{\theta_{\mathrm{r}}+\kappa_{\mathrm{r}}} \mathrm{d} \theta_{\mathrm{r}} \int_{\varphi_{i}-\alpha\left(\theta_{\mathrm{i}}\right)}^{\varphi_{\mathrm{i}}+\alpha\left(\theta_{\mathrm{i}}\right)} \mathrm{d} \varphi_{\mathrm{i}} \int_{\varphi_{\mathrm{r}} 0-\alpha\left(\theta_{\mathrm{r}}\right)}^{\left.\varphi_{\mathrm{r}}+\alpha \theta_{\mathrm{r}}\right)} \mathrm{d} \varphi_{\mathrm{r}} \int_{0}^{2 \pi} \mathrm{d} \gamma \int_{0}^{R_{\mathrm{A}}} \mathrm{dr} F}{\pi \sin ^{2} \kappa_{\mathrm{i}} \cos \theta_{\mathrm{i} 0}}
\end{aligned}
$$

where

$$
F=r \cos \theta_{\mathrm{i}} \sin \theta_{\mathrm{i}} \cos \theta_{\mathrm{r}} \sin \theta_{\mathrm{r}} S\left(\theta_{\mathrm{i}}, \varphi_{\mathrm{i}} ; \theta_{\mathrm{r}}, \varphi_{\mathrm{r}}\right)
$$

and

$\alpha\left(\theta_{\mathrm{n}}\right)=\cos ^{-1}\left(\frac{\cos \kappa_{\mathrm{n}}-\cos \theta_{\mathrm{n}} \cos \theta_{\mathrm{n} 0}}{\sin \theta_{\mathrm{n}} \sin \theta_{\mathrm{n} 0}}\right)$

with $n=i$ or $r$.

$R_{\mathrm{A}}$ is the radius of the measurement area, $r$ and $\gamma$ are, respectively, the radial and polar coordinates used to integrate over the measurement area, $\kappa_{\mathrm{i}}$ and $\kappa_{\mathrm{r}}$ are the angles subtended by the irradiation and collection apertures (see Fig. 2), and $\theta$ and $\varphi$, respectively, refer to the polar and azimuth spherical coordinates defining corresponding directions denoted as $\mathbf{r}$. Notice that the angles subtended by the measurement area $\kappa_{\mathrm{ma}, \mathrm{i}}$ and $\kappa_{\text {ma,r }}$ (see Fig. 2) are used in the area integral $\int_{0}^{2 \pi} \mathrm{d} \gamma \int_{0}^{R_{\mathrm{A}}} \mathrm{dr} F$.

\section{Evaluation of the measured BRDF for different conditions}

In order to evaluate the impact of finite intervals in the BRDF measurement for non-Lambertian surfaces, the relative deviation of the measured BRDF [estimated by equation (11)] from the actual BRDF $\left(f_{\mathrm{r}}\right)$ was examined for different conditions. For this purpose, a family of bidirectional scattering-surface reflectance distribution functions $(S)$ was defined, with a very simple dependence on $\mathbf{r}_{\mathrm{i}}$ and $\mathbf{r}_{\mathrm{r}}$. This family provides a wide range of curvatures and includes angular scattering distribution functions from Lambertian to highly specular with high second derivatives (large curva- tures). These functions are therefore adequate to study the measured BRDF around specular peaks, which is an especially challenging case. ${ }^{5}$ The family of curves that represents $S$ can be defined as:

$S\left(\beta, \theta_{\text {asp }}\right)=\beta e^{-\beta \theta_{\text {asp }}}$

where $\theta_{\text {asp }}$ is the aspecular angle, or angular deviation between collection and specular directions $\left[\cos ^{-1}(\cos \right.$ $\left.\theta_{\mathrm{i} 0} \cos \theta_{\mathrm{r} 0}+\sin \theta_{\mathrm{i} 0} \sin \theta_{\mathrm{r} 0} \sin \varphi_{\mathrm{r} 0}\right)$, always positive], and $\beta$ is a free parameter. Notice that the larger the $\beta$, the larger is the curvature too, and that $\beta \rightarrow 0$ represents the Lambertian distribution. Different values of $\beta$ and $\theta_{\text {asp }}$ define different curvatures of $S$. For instance, a very low value of $\beta$ produces an almost constant value of $S$ around high values of $\theta_{\text {asp }}$, whereas very high curvatures are obtained by combining high values of $\beta$ and low values of $\theta_{\text {asp. }}$.

Four functions of this family $(\beta=1 / 2, \beta=1 / 4, \beta=1 /$ 8 and $\beta=1 / 16$, with units of degrees ${ }^{-1}$ ) are shown in Fig. 3. In the literature ${ }^{5}$, it is claimed that glossy surfaces can be encountered with very narrow BRDF, with typical full width at half maximum (FWHM) inferior to $0.5^{\circ}$, which correspond to $\beta$ values larger than 3. However, until now, conventional goniospectrophotometers $^{7-12}$ do not allow those peaks to be resolved with metrological accuracy. Only special conoscopic systems ${ }^{5}$ have been proved adequate to achieve that angular resolution, and they require a separate discussion. We think that the curvatures given by the selected functions are enough to model most of the experimental conditions. In the case of highly glossy samples, there may be a minimum aspecular

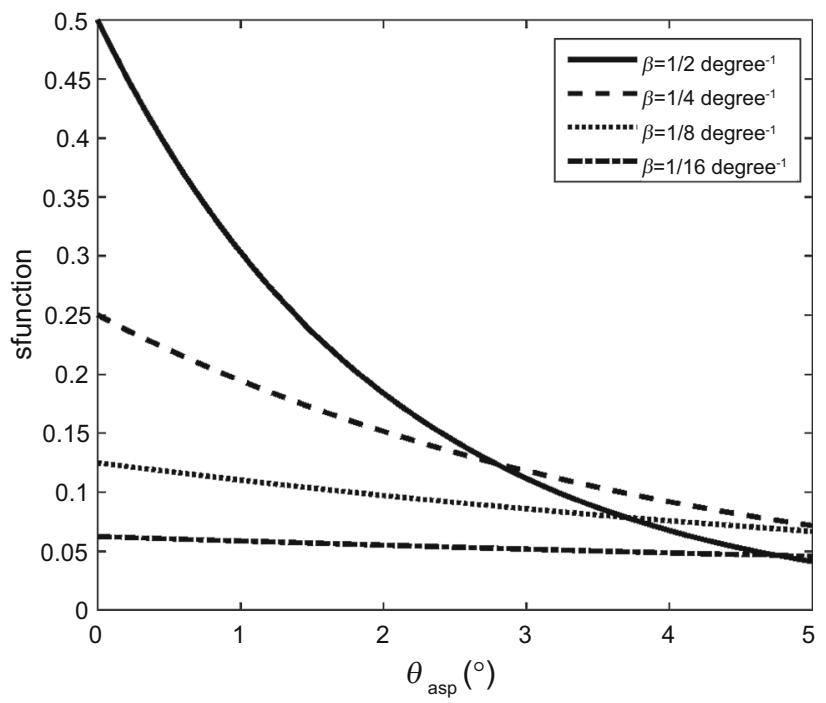

Fig. 3: Examples of bidirectional scattering-surface reflectance distribution functions used in the evaluation of the measured BRDF $(\beta=1 / 2, \beta=1 / 4, \beta=1 / 8$, and $\beta=1 /$ 16 , with units of degrees ${ }^{-1}$ ) 
angle from which the results of this work cannot be applied.

To understand the impact of finite angles, these representative scattering functions were used along with different realistic geometrical conditions, with combinations of the subtended angles $\kappa_{\mathrm{i}}, \kappa_{\mathrm{r}}, \kappa_{\mathrm{ma}, \mathrm{i}}$, and $\kappa_{\text {ma,r }}$ (as defined in Fig. 2). To reduce the analysis, a negligible value of $\kappa_{\mathrm{i}}$ was used $\left(0.0006^{\circ}\right.$, very small irradiation aperture), which means that the effect produced by the values of $\kappa_{\mathrm{r}}$ prevails over the effect produced by $\kappa_{\mathrm{i}}$. There is reciprocity between $\kappa_{\mathrm{i}}$ and $\kappa_{\mathrm{r}}$ since, according to the Helmholtz reciprocity principle, the result from the analysis is equal to the inverted case at which apertures and distances are interchanged between source and collector, as long as the measurement is for unpolarized reflectance ${ }^{33}$. Values of $\kappa_{\mathrm{r}}$, $\kappa_{\mathrm{ma}, \mathrm{i}}$, and $\kappa_{\mathrm{ma,r}}$ were selected as $0.11^{\circ}, 0.23^{\circ}, 0.49^{\circ}$, or $0.92^{\circ}$, with the additional condition that $\kappa_{\mathrm{ma}, \mathrm{i}}$ is always equal to $\kappa_{\mathrm{ma}, \mathrm{r}}$, equivalent to considering that the selected distances from source and receiver to the measurement area are identical. The selected $\kappa$ values are realistic. If source and detector were located at a distance of $1 \mathrm{~m}$ from the sample, the diameter of their areas (assumed circular) would be $3.8 \mathrm{~mm}$ for the minimum $\left(0.11^{\circ}\right)$ and $32.1 \mathrm{~mm}$ for the maximum $\left(0.92^{\circ}\right)$. It must be reminded here that the solid angles subtended by the measurement area are projected under different incidence and collection directions and that the values of $\kappa_{\mathrm{ma}, \mathrm{i}}$ and $\kappa_{\mathrm{ma}, \mathrm{r}}$ reported in this work are those at normal irradiation or collection directions.

The measured BRDF was calculated using equation (11) for the four functions shown in Fig. 3, and for the different geometries, as described in the above paragraph.

The complete evaluation was carried out at two irradiation angles $\left(\theta_{\mathrm{i} 0}=20^{\circ}\right.$ and $\left.\theta_{\mathrm{i} 0}=70^{\circ}\right)$, and for those aspecular angles for which the function is $90 \%$ of its maximum value $\left(\theta_{\text {asp,90 }}\right)$. At these aspecular angles, the curvature of the reflectance is $90 \%$ of the maximum curvature observed in the sample. The deviations, calculated as the relative difference between the actual BRDF and the estimated measured BRDF, are shown in Fig. 4. Each subplot corresponds to a different surface, with a BRDF calculated at different values of the parameter $\beta$, and, consequently, described with a different value of $\theta_{\text {asp.90. }}$. It is observed that this deviation is always larger for lower irradiation angles, for larger $\kappa$ values, and for larger $\beta$ values, if the other involved variables are fixed. The awkward behavior of the two lower lines in the lower left plot is precision artifacts from the numerical calculation.

As expected, the larger the $\beta$ (related to sample's scattering curvature) and $\kappa$ values (subtended angles) are, the larger the deviation is. The relative deviation obtained from the evaluation is similar for the two selected irradiation angles, but slightly lower for the largest one. (Note that different irradiation angles are represented with lines of different thicknesses in Fig. 4.) The reason for this difference is that at larger angles the incidence angles $\theta_{\mathrm{i}}$ on the measurement area are more closely distributed around the irradiation angle $\theta_{\mathrm{i} 0}$, since the solid angle subtended by this area is projected.

This relative deviation is represented as a function of $\kappa_{\mathrm{r}}$, and every curve corresponds to a different value of $\kappa_{\mathrm{ma}, \mathrm{i}}$, which is equal to $\kappa_{\mathrm{ma}, \mathrm{r}}$ in this case. The curves are constant when the effect of $\kappa_{\mathrm{ma}, \mathrm{r}}$ dominates that of $\kappa_{\mathrm{r}}$.

\section{General rule to estimate error}

The error for any condition can be estimated as the difference between $f_{\mathrm{r}}$ and the result from equation (11). However, it may be of interest for the experimentalist to have a general rule or metric to estimate errors. The described methodology was used to obtain the following general rule to calculate the error introduced by finite intervals when measuring BRDFs varying with the incidence and collection directions within a small angular range. Equations (11)-(13) were used to estimate the "measured" BRDF under different measuring conditions of surfaces with the scattering distribution functions described by equation (14).

An estimation of a "measured" BRDF is defined by $\beta, S / S_{\max }\left[\right.$ or $\left.S\left(\beta, \theta_{\text {asp }}\right) / S(\beta, 0)\right], \theta_{\mathrm{i} 0}, \kappa_{\mathrm{i}}, \kappa_{\mathrm{r}}, \kappa_{\mathrm{ma}, \mathrm{i}}$, and $\kappa_{\mathrm{ma}, \mathrm{r}}$. Notice that $\theta_{\text {asp }}$ can be determined from $\beta$ and $S /$ $S_{\text {max }}\left(\theta_{\text {asp }}=-\ln \left(S / S_{\max }\right) / \beta\right)$ and that $\theta_{\mathrm{r} 0}$ can be obtained from the values of $\theta_{\text {asp }}$ and $\theta_{\mathrm{i} 0}$. In order to assess the impact of the different variables on the error due to finite intervals, two sets of estimations were defined, both of them for $\beta=1 / 2$ degrees $^{-1}, \beta=1 / 4$ degrees $^{-1}$, $\beta=1 / 8$ degrees $^{-1}$, and $\beta=1 / 16$ degrees $^{-1}$. The motivation of this strategy was to make the analysis more efficient in terms of computational time, while providing the most valuable information. Note that each estimation is the sum of around 50 millions terms used to calculate the integral in equation (11) with a reduced level of noise and that the calculation of the second set of estimations described below took $80 \mathrm{~h}$.

The first set of estimations assessed the sensitivity of the error to $S / S_{\max }$, which is closely related to the curvature of $S$. This value was selected from 0.25 to 0.95 , with steps of 0.05 . The variable $\theta_{\mathrm{i} 0}$ and the relation between the variables $\kappa_{\mathrm{i}}, \kappa_{\mathrm{r}}, \kappa_{\mathrm{ma}, \mathrm{i}}$, and $\kappa_{\mathrm{ma}, \mathrm{r}}$ were fixed $\left(\theta_{\mathrm{i} 0}=5^{\circ}\right.$, and $\kappa_{\mathrm{i}}: \kappa_{\mathrm{r}}: \kappa_{\mathrm{ma}, \mathrm{i}}: \kappa_{\mathrm{ma}, \mathrm{r}}=$ $0.25: 1: 0.5: 1)$, as described in the second column of Table 1. Values of $\kappa_{\mathrm{r}}=0.23^{\circ}, 0.46^{\circ}, 0.92^{\circ}$, and $1.83^{\circ}$ were selected.

The second set of estimations was defined to assess the variation of the error at different values of $\theta_{\mathrm{i} 0}$ and to confirm the relationships observed after examining the estimations of the first set. In this set (see third column of Table 1), fewer values of $S / S_{\max }$ were used $(0.3,0.6$, and 0.9$) . \theta_{\mathrm{i} 0}$ ran from $15^{\circ}$ to $75^{\circ}$, with steps of $15^{\circ}$, and estimations were done for all combinations of the values $0.11^{\circ}, 0.34^{\circ}$, and $0.92^{\circ}$ for $\kappa_{\mathrm{i}}, \kappa_{\mathrm{r}}, \kappa_{\mathrm{ma}, \mathrm{i}}$, and $\kappa_{\text {ma,r }}$.

Insight into the results of the calculations and the steps followed in the analysis is given in the two plots 

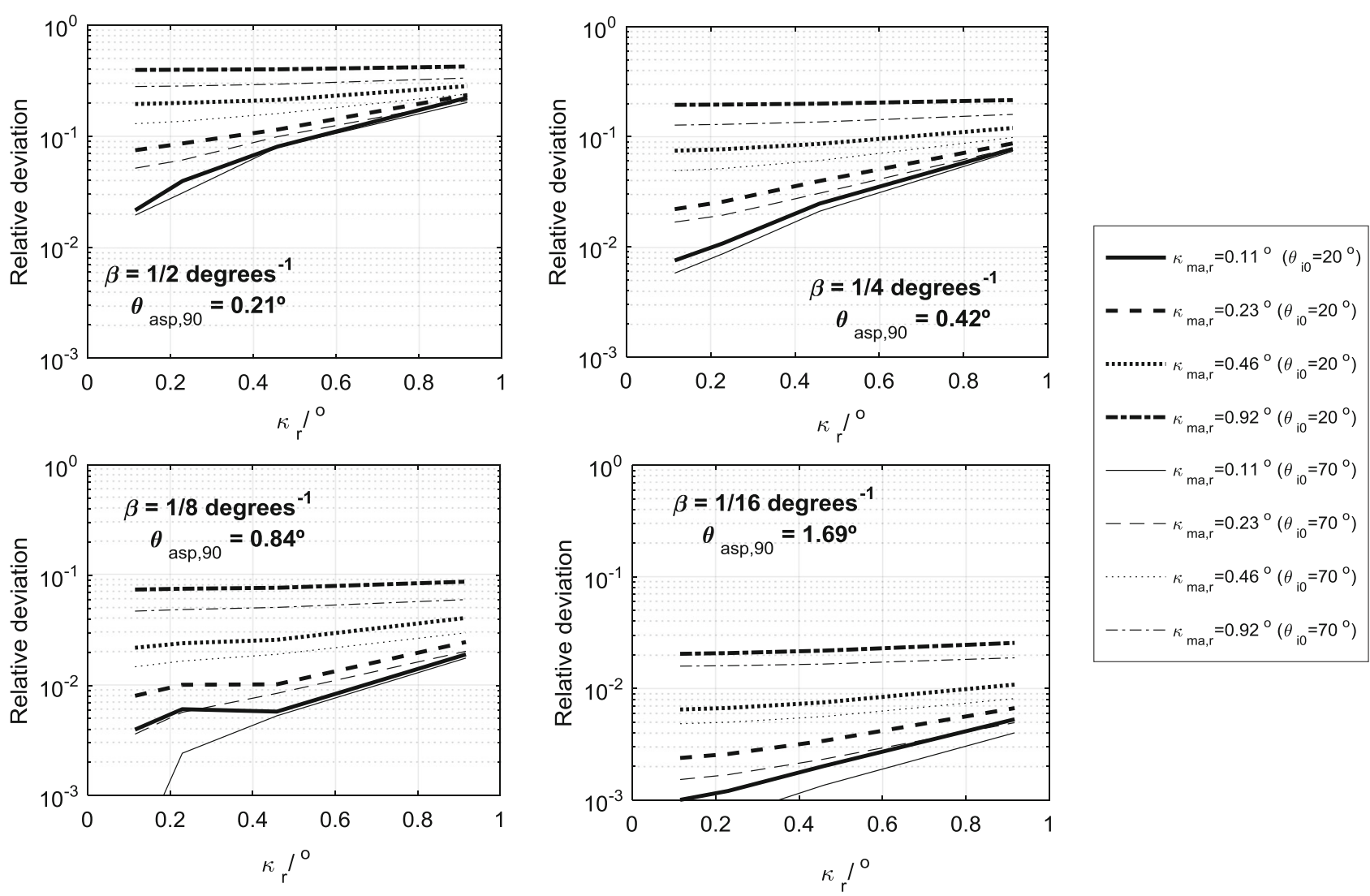

Fig. 4: Relative deviations with respect to the actual BRDF at the corresponding aspecular angle $\theta_{\mathrm{asp}, 90}$, and for $\theta_{\mathrm{i} 0}=20^{\circ}$ and $\theta_{\mathrm{i} 0}=70^{\circ}$

Table 1: Description of the two sets of estimations used to assess the impact of the different variables on the error due to finite intervals

Variables

Set 1 of estimations

Set 2 of estimations

\begin{tabular}{|c|c|c|}
\hline$\theta_{\mathrm{i} 0}$ & $5^{\circ}$ & From $15^{\circ}$ to $75^{\circ}\left(\Delta=15^{\circ}\right)$ \\
\hline$S / S_{\max }$ & From 0.25 to $0.95(\Delta=0.05)$ & $0.3,0.6,0.9$ \\
\hline$\kappa_{\mathrm{i}}, \kappa_{\mathrm{r}}, \kappa_{\mathrm{ma}, \mathrm{i}}, \kappa_{\mathrm{ma}, \mathrm{r}}$ & $\begin{array}{l}\kappa_{\mathrm{r}}=0.23^{\circ}, 0.46^{\circ}, 0.92^{\circ}, 1.83^{\circ} \\
\text { Relation } \kappa_{\mathrm{i}}: \kappa_{\mathrm{r}}: \kappa_{\mathrm{ma}, \mathrm{i}}: \kappa_{\mathrm{ma}, \mathrm{r}}=0.25: 1: 0.5: 1\end{array}$ & All combinations with any $\kappa$ taking $0.11^{\circ}, 0.34^{\circ}$, and $0.92^{\circ}$ \\
\hline $\begin{array}{l}\text { Number of } \\
\text { estimations }\end{array}$ & 240 & 1620 \\
\hline
\end{tabular}

shown in Fig. 5. In the upper plot, the relative errors of the two sets of estimations are represented as a function of $S$. The data of the second set of estimations are arranged in nine vertical lines, which correspond with the nine $S$ values that were obtained for this set [see attainable values for $S=\left(S / S_{\max }\right) \times \beta$ ]. The data of the first set are scattered uniformly over the entire $S$ range, but are somewhat arranged too on four curves. The estimated errors are related to the value of $S$ because they are related to curvature and curvature is higher for higher $S$. But it is not the only variable that affects the error, and that is the reason for the four observed curves and the data scattering.
In the lower plot, the estimated errors of the two sets are again represented, but in this case as a function of $S \times F_{\kappa}$, where $F_{\kappa}$ is a factor including the finite intervals. It is defined as:

$F_{\kappa}=\left(\kappa_{\mathrm{i}}+\kappa_{\mathrm{ma}, \mathrm{i}}+\kappa_{\mathrm{r}}+\kappa_{\mathrm{ma}, \mathrm{r}}\right)^{0.9}$

with the $\kappa$ values expressed in degrees. There is a clear relation between $S \times F_{\kappa}$ and the relative error due to finite intervals combined with the curvature growth with $\mathrm{S}$ value.

By using the 1860 estimations, it was possible to obtain a function expressing the dependence of the 

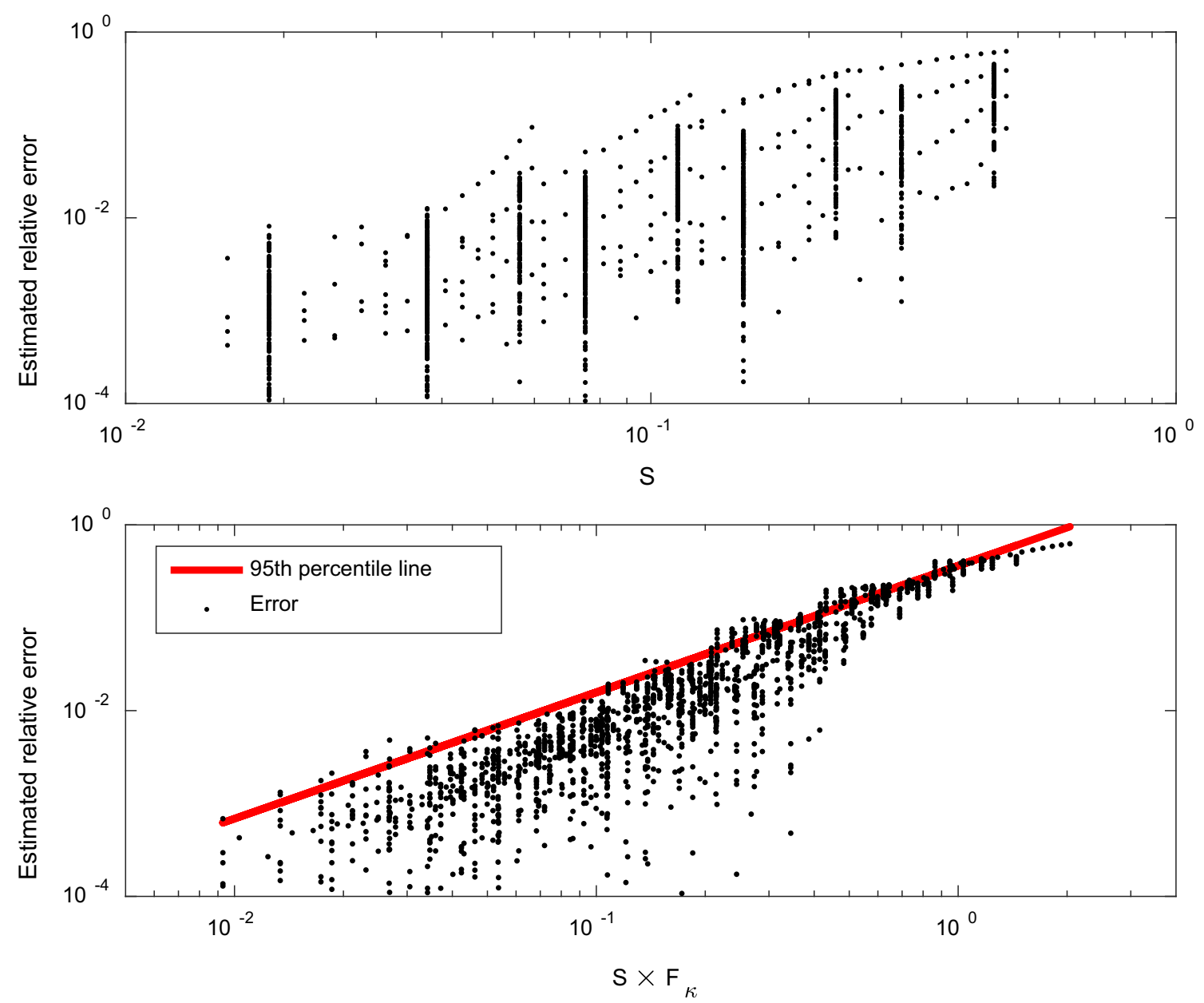

Fig. 5: (Upper plot): Estimated errors of the two sets of estimations as a function of $S$. (Lower plot): Estimated errors as a function of $S \times F_{k}$

95th percentile of the estimated error on $S \times F_{\kappa}$. After trying different types of functions, it was found that an exponential function was the most adequate to relate estimated errors to $S \times F_{\kappa}$. The values of $S$ used in the fitting were calculated as the product of the parameters $\left(S / S_{\max }\right)$ and $\beta$ (see Table 1$)$. The equation resulting from the fitting is given below in equation (18). This function is shown in the lower plot of Fig. 5 as a continuous line. This function allows the 95th percentile of the relative error $\left[\delta_{95}\left(f_{\mathrm{r}}\right)\right]$ to be estimated from the $\kappa$ values and the curvature of $S$, and it can be used as metric for this kind of error.

The 95th percentile of error function was obtained using a specific family of scattering distribution functions $S$. Since it is not obvious that this result can be applied to other functions, it must be clarified here how to apply this function to practical cases. Notice that the function in equation (14) is defined in such a way that the larger its value, the larger its curvature, and that is why $\delta_{95}\left(f_{\mathrm{r}}\right)$ is proportional to $S\left(\beta, \theta_{\text {asp }}\right)$ for this family of functions for a given instrument with constant $F_{\kappa}$. This is not necessarily the case for other functions, but, within a small range of an angular variable $\theta$, an exponential function:

$S=A \times \beta \times e^{-\beta \times\left|\theta-\theta_{0}\right|}$

as in equation (14), can model any reflectance data with monotonic concave curvature, where the parameter $A$ can be regarded as a scale constant which does not affect the estimated error, and $\beta$ and $\theta_{0}$ are other free parameters. For monotonic reflectance data with convex curvature, the convex function

$S=A \times \beta \times\left[2 e^{-\beta \times\left|\theta_{\mathrm{m}}-\theta_{0}\right|}-e^{-\beta \times\left|\theta-\theta_{0}\right|}\right]$

( $\theta_{\mathrm{m}}$ being the measurement angle) can be used as model, which is symmetric to the previous one with respect to $S=S\left(\theta_{\mathrm{m}}\right)$ and produces the same value of $\delta_{95}\left(f_{\mathrm{r}}\right)$.

Once the parameters $\beta$ and $\theta_{0}$ are obtained, $\delta_{95}\left(f_{\mathrm{r}}\right)$ can be estimated for any surface using the function for the 95th percentile of error found above, as: 
${ }_{95}\left(f_{\mathrm{r}}\right)=0.36 \times\left[\beta \times e^{-\beta \times\left|\theta-\theta_{0}\right|} \times F_{\kappa}\right]^{1.36}$

This expression is obtained from the fit shown in the lower plot in Fig. 5. The value of $\beta$ must be given in degree $^{-1}$, and the values of the different $\kappa \mathrm{s}$, and any angular variable, in degrees.

\section{Discussion}

Equations (16)-(18) developed from the simulations described above can be used to evaluate the value of the 95th percentile of the relative finite-intervalsrelated error for a particular experimental setup. Equation (18) is composed of two important factors: $\beta \times e^{-\beta \times\left|\theta-\theta_{0}\right|}$ and $F_{\kappa}$ which are, respectively, related to the scattering distribution function of the surface and the size of the finite intervals. Any of these two factors can make the error negligible if it is small enough, no matter the value of the other factor. For instance, a small $\beta$, which makes the first factor very small, represents a Lambertian surface and produces a negligible error regardless of the size of the finite intervals (the values of $\kappa$ ). On the other hand, making $\kappa$ values small enough, the committed error can be made negligible even in the extreme case of very large values of $\beta \times e^{-\beta \times\left|\theta-\theta_{0}\right|}$, at geometries close to specular peaks.

In general, keeping similar values for $\kappa_{\mathrm{i}}$ and $\kappa_{\mathrm{ma}, \mathrm{i}}$ (and for $\kappa_{\mathrm{r}}$ and $\kappa_{\mathrm{ma}, \mathrm{r}}$ ) is optimal in a goniospectrophotometer, since a lower value of only one of these variables does not reduce significantly the relative error due to finite intervals, and reduces the available light, which increases other uncertainty sources.

Equation (18) supports design or characterization of goniospectrophotometers or multiangle spectrophotometers, since it allows the applicability range of the instrument to be specified for a target uncertainty. For instruments with a fixed value of $F_{\kappa}$, as it is the case of commercially available portable multiangle spectrophotometers, it may help to estimate for different types of samples the finite intervals uncertainty at their measurement geometries, which usually have different aspecular angles. Aspecular angles would be used as $\left|\theta-\theta_{0}\right|$ value in equation (18), and different values for $\beta$ would be used to illustrate different types of samples, from very diffuse to very glossy. Instruments allowing the variation of the size of apertures, and consequently $F_{\kappa}$, can be adapted to the specific type of sample to be measured. For these instruments, the necessary $F_{\kappa}$ is worked out from equation (18), using a value of $\beta$ which, based on the experience, corresponds with the observed glossiness of the sample, and, as $\delta_{95}$, the aimed maximum relative uncertainty. Those instruments designed to measure at the specular peak, as glossimeters, introduce an error which depends on their apertures. In such cases, that error cannot be estimated by equation (18), but by comparing the result from equation (11) with the BRDF. To illustrate the error for different types of samples, the BRDF can be modeled with the family of curves in equation (14). In general, equation (11) is recommended to simulate complex conditions for which the derived equation (18) is not suitable.

To estimate experimentally the relative error for a given specimen, the values of $\beta$ and $\theta_{0}$ must be known for every measurement geometry. If the expected error due to finite intervals is relatively low, the "measured" BRDF itself can be used to estimate $\beta$ and $\theta_{0}$. However, if a high error is expected in the measurement, as for low aspecular angles in highly glossy specimens, some deconvolution algorithm needs to be applied to estimate $\beta$ and $\theta_{0}$ from the deconvolved BRDF. $^{14,15}$ In these cases, equation (18) is recommended to be used to characterize the limitations of the instrument to measure BRDFs with high angular dependence, and to redesign it if required.

It must be noticed that there are limitations in the applicability of equation (18), since it was derived for a range of curvature and $\kappa$ values. The maximum curvature used in the derivation was slightly larger than 0.1 , so that it can be recommended to use equation (18) only when it is expected that:

curvature $: \frac{\frac{\mathrm{d}^{2} S}{\mathrm{~d} \theta^{2}}}{\left|1+\left(\frac{d S}{d \theta}\right)^{2}\right|^{3 / 2}}=\frac{\beta^{3} e^{-\beta\left|\theta-\theta_{0}\right|}}{\left|1+\beta^{4} e^{-2 \beta\left|\theta-\theta_{0}\right|}\right|^{3 / 2}} \leq 0.1$

with $\theta_{0}$ and $\beta$ obtained from equations (16) or (17).

Regarding $\kappa$ values, the use of equation (18) is restricted to values below $1^{\circ}$.

\section{Conclusions}

The impact of using finite intervals (for the measurement area, irradiation and collection apertures, and solid angles) has been examined for the measurement of BRDF. A theoretical approach has been proposed to evaluate the limitations of experimental systems for the measurement of the BRDF and to account for the relative error exclusively due to finite intervals. This error, calculated as the relative difference between a biconical integration of the BRDF and the actual BRDF, is composed of two main factors, which are related to the scattering distribution function of the surface, and the geometries of the incident and collected radiant fluxes. Any of these factors can reduce to negligible the total error if it is set to a value small enough. The general rule for experimentalists provided here should be useful for the design of instruments for measuring BRDFs very dependent on incidence and collection geometries, such as those intended to measure gloss or iridescence. 
Acknowledgments This article was written within the EMPIR 16NRM08 Project BiRD "Bidirectional reflectance definitions." The EMPIR is jointly funded by the EMPIR participating countries within EURAMET and the European Union. The author is also grateful to Comunidad de Madrid for funding the project SINFOTON-CM: S2013/MIT-2790.

Open Access This article is distributed under the terms of the Creative Commons Attribution 4.0 International License (http://creativecommons.org/lice nses/by/4.0/), which permits unrestricted use, distribution, and reproduction in any medium, provided you give appropriate credit to the original author(s) and the source, provide a link to the Creative Commons license, and indicate if changes were made.

Funding The funding for this was obtained from EURAMET and the European Union (16NRM08 Project BiRD), and Comunidad de Madrid (S2013/ MIT-2790).

\section{References}

1. Nicodemus, FE, "Directional Reflectance and Emissivity of an Opaque Surface.” Appl. Opt., 4 767-775 (1965)

2. Nicodemus, FE, Richmond, JC, Hsia, JJ, "Geometrical Considerations and Nomenclature for Reflectance." Natl. Bur. Stand. Monogr. (1977). https://doi.org/10.1109/LPT.200 9.2020494

3. Judd, DB, "Terms, Definitions, and Symbols in Reflectometry." JOSA, 57 445-452 (1967)

4. Ferrero, A, Rabal, AM, Campos, J, Pons, J, Hernanz, ML, "Spectral and Geometrical Variation of the Bidirectional Reflectance Distribution Function of Diffuse Reflectance Standards." Appl. Opt., 51 8535-8540 (2012)

5. Obein, G, Audenaert, J, Ged, G, Leloup, FB, "Metrological Issues Related to BRDF Measurements Around the Specular Direction in the Particular Case of Glossy Surfaces."
Proc. SPIE 9398, Measuring, Modeling, and Reproducing Material Appearance 2015, 93980D, 13 March 2015

6. McCamy, C, "Concepts Terminology and Notation for Optical Modulation." Photogr. Sci. Eng., 10314 (1966)

7. Obein, G, Bousquet, R, Nadal, ME, "New NIST Reference Goniospectrometer." Proc. SPIE 5880, Optical Diagnostics, 58800T, 24 August 2005

8. Rabal, AM, Ferrero, A, Campos, J, Fontecha, JL, Pons, A, Rubiño, AM, Corróns, A, "Automatic Goniospectrophotometer for the Absolute Measurement of the Spectral BRDF at In-and Out-of-Plane and Retroreflection Geometries." Metrologia, 49 213-223 (2012)

9. Hünerhoff, D, Grusemann, U, Höpe, A, "New Robot-Based Gonioreflectometer for Measuring Spectral Diffuse Reflection." Metrologia, 43 S11-S16 (2006)

10. Leloup, FB, Forment, S, Dutré, P, Pointer, MR, Hanselaer, $\mathrm{P}$, "Design of an Instrument for Measuring the Spectral Bidirectional Scatter Distribution Function." Appl. Opt., 47 5454-5467 (2008)

11. Baribeau, R, Neil, WS, Côté, E, "Development of a RobotBased Gonioreflectometer for Spectral BRDF Measurement." J. Mod. Opt., 56 1497-1503 (2009)

12. Matsapey, N, Faucheu, J, Flury, M, Delafosse, D, "Design of a Gonio-Spectrophotometer for Optical Characterization of Gonio-Apparent Materials." Meas. Sci. Technol., 24065901 (2013)

13. Clarke, F, Parry, D, "Helmholtz Reciprocity: Its Validity and Application to Reflectometry." Light. Res. Technol., 17 1-11 (1985)

14. Ferrero, A, Campos, J, Rabal, AM, Pons, A, Hernanz, ML, Corróns, A, "Deconvolution of Non-Zero Solid Angles Effect in Bidirectional Scattering Distribution Function Measurements." Proc. SPIE 8083, Modeling Aspects in Optical Metrology III, 808315, 23 May 2011

15. Audenaert, J, Leloup, FB, Durinck, G, Deconinck, G, Hanselaer, P, "Bayesian Deconvolution Method Applied to Experimental Bidirectional Transmittance Distribution Functions." Meas. Sci. Technol., 24035202 (2013)

Publisher's Note Springer Nature remains neutral with regard to jurisdictional claims in published maps and institutional affiliations. 